\title{
Imaging features of primary spinal osseous tumors and their value in clinical diagnosis
}

\author{
LILI YANG ${ }^{1}$, SHUQUAN ZHANG ${ }^{2}$, RUI GU ${ }^{3}$, CHUANGANG PENG $^{1}$ and MINFEI WU ${ }^{1}$ \\ ${ }^{1}$ Department of Orthopaedics, The Second Hospital of Jilin University, Changchun, Jilin 130041; \\ ${ }^{2}$ Department of Orthopaedics, Nankai Hospital, Tianjin 300100; \\ ${ }^{3}$ Department of Orthopaedics, China-Japan Union Hospital of Jilin University, Changchun, Jilin 130033, P.R. China
}

Received March 27, 2018; Accepted September 28, 2018

DOI: $10.3892 / \mathrm{ol} .2018 .9659$

\begin{abstract}
This study explored the method of imaging diagnosis of primary spinal osseous tumors and the application value of imaging in clinical diagnosis. Sixty-nine patients with primary spinal osseous tumors who received treatment in Nankai Hospital from July 2016 to June 2017 were selected. All of them received X-ray, computed tomography (CT) and magnetic resonance imaging (MRI) examinations to analyze the imaging features of the three examination methods. Sensitivity (Sen), specificity (Spe), positive predictive value $\left(\mathrm{PV}^{+}\right)$, negative predictive value $\left(\mathrm{PV}^{-}\right)$and accuracy (Acc) were compared. The consistency of the three examination methods in diagnosing primary spinal osseous tumors was analyzed. Sen, Acc and $\mathrm{PV}^{-}$of the three examination methods in diagnosing spinal osseous tumors had obvious differences. MRI showed the highest $\operatorname{Sen}(\mathrm{P}<0.05)$. MRI had relatively high consistency with CT scan in diagnosing primary spinal osseous tumors, and $\kappa$-value was 0.72 . CT scan and X-ray had obvious difference in diagnosing primary spinal osseous tumors $(\mathrm{P}<0.05)$. The consistency between CT scan and X-ray in diagnosing primary spinal osseous tumors was relatively low, and $\kappa$-value was 0.47 . MRI and X-ray had obvious difference in diagnosing primary spinal osseous tumors $(\mathrm{P}<0.05)$. The consistency between MRI and X-ray in diagnosing primary spinal osseous tumors was relatively low, and $\kappa$-value was 0.41 . X-ray examination is easy to operate with high resolution. CT scan has obvious advantages in displaying lesions with complex structure, many of which locate in overlapping sites. MRI has more advantages and higher accuracy in judging the scope of the tumor. CT and MRI examinations have obviously higher efficacy than X-ray in diagnosing primary spinal osseous tumors. They are conducive in improving the accuracy of diagnosing primary spinal osseous tumors.
\end{abstract}

Correspondence to: Dr Minfei Wu, Department of Orthopaedics, The Second Hospital of Jilin University, 126 Ziqiang Street, Changchun, Jilin 130041, P.R. China

E-mail: wumingfei999@163.com

Key words: primary spinal osseous tumor, imaging, diagnosis

\section{Introduction}

Spinal osseous tumors have complex lesion types, which can be divided into metastatic tumor and primary tumor, in which primary spinal osseous tumor is a rare tumor disease taking up approximately $7 \%$ in systemic tumors, and its rate of deterioration is relatively high with the occurrence of disease $(1,2)$. The highest proportion of metastatic tumor is $>50 \%$, half of which are benign tumors and the remaining half are malignant tumors. It is characterized by various types, low differences in imaging features and difficult diagnosis. As the nerves and capillaries are densely distributed around the spine, removal of the tumor with minimal wound can be reached only after the occurrence site and the scope of the tumor are mastered well (3). Therefore, in order to reduce misdiagnosis and postoperative recurrence rates, a total of 69 patients with primary spinal osseous tumors were analyzed to further explore the imaging features and provide guidance for clinical diagnosis and treatment.

\section{Patients and methods}

General patient data. A total of 69 patients with primary spinal osseous tumors who received treatment in Nankai Hospital (Tianjin, China) from July 2016 to June 2017 were selected into the study. It was confirmed by pathological diagnosis that all the patients suffered from the disease, and all of them received X-ray, computed tomography (CT) and magnetic resonance imaging (MRI) examinations (4). General data of study patients are given in Table I. The study was approved by the Ethics Committee of Nankai Hospital and informed consents were signed by the patients or guardians.

$X$-ray examination. X-ray examination was conducted for all the patients. Siemens digital radiography (DR) machine was adopted for X-ray on the posteroanterior and single lateralposition of the pain site of the patient. X-ray was conducted on double oblique positions if necessary.

CT examination. All the patients received CT examinations in a supine position using a dual-source 64-row CT scanner (produced by Siemens, Munich, Germany). Parameters of the CT scanner were set as follows. Current: 260-300 msec; 
Table I. General data of the patients.

\begin{tabular}{lc}
\hline Items & Subject $(\mathrm{n}=69)$ \\
\hline Male (n, \%) & $36(52.17)$ \\
Female (n, \%) & $33(47.83)$ \\
Age (years) & $10-70$ \\
Average age (years) & $24.36 \pm 4.49$ \\
Clinical manifestation & \\
Chest and back pain & $52(75.36)$ \\
Radioactive pain & $14(20.29)$ \\
Fever & $19(27.54)$ \\
Local swelling & $5(7.25)$ \\
Perspiration dysfunction & $11(15.94)$ \\
Limited mobility & $3(4.35)$ \\
\hline
\end{tabular}

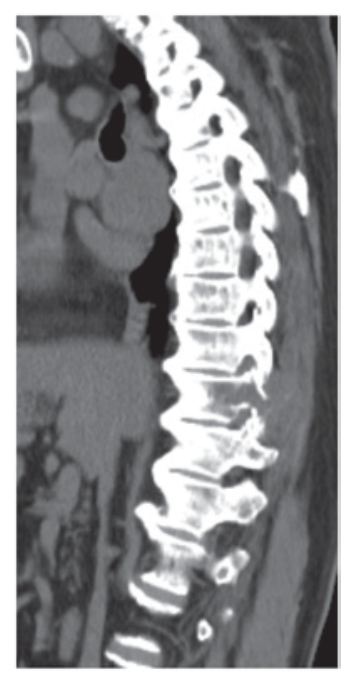

Figure 1. A CT scan on a female patient with osteochondroma aged 42 years shows that the boundary is blurry and irregular, and that calcification is generated in the lesion.

voltage: $120 \mathrm{kV}$; layer thickness: $5 \mathrm{~mm}$; interlayer spacing: $1 \mathrm{~mm}$; matrix: $512 \times 512$. The scanning was conducted on the spine with lesions and the adjacent vertebral body. An enhanced scan was conducted with iohexol [produced by Guangzhou Schering Pharmaceutical Co., Ltd., Guangzhou, China, concentration: $300 \mathrm{mg} \mathrm{I} / \mathrm{ml}$ ], a non-ionic iodinecontaining contrast agent, in a dose of $1.5 \mathrm{ml} / \mathrm{kg}$ (body weight) by bolus injection with a high-pressure syringe in the same method as plain scan.

MRI examination. All the patients received MRI examinations with an MRI scanner (MR 3.0T HDX TWINSP produced by General Electric Co., Fairfield, CT, USA). Routine T1 weighted imaging (T1WI) and T2 weighted imaging (T2WI) examinations were conducted, in which T1WI: time of repetition (TR): 200-540 msec, time to echo (TE): 2-14 msec; T2WI: $\mathrm{TR}=3,000-5,000 \mathrm{msec}, \mathrm{TE}=23-138 \mathrm{msec}$. The scanning site was the center of the patient's pain site, and the scope of the scanning was the spine and the adjacent vertebral body of the patient.

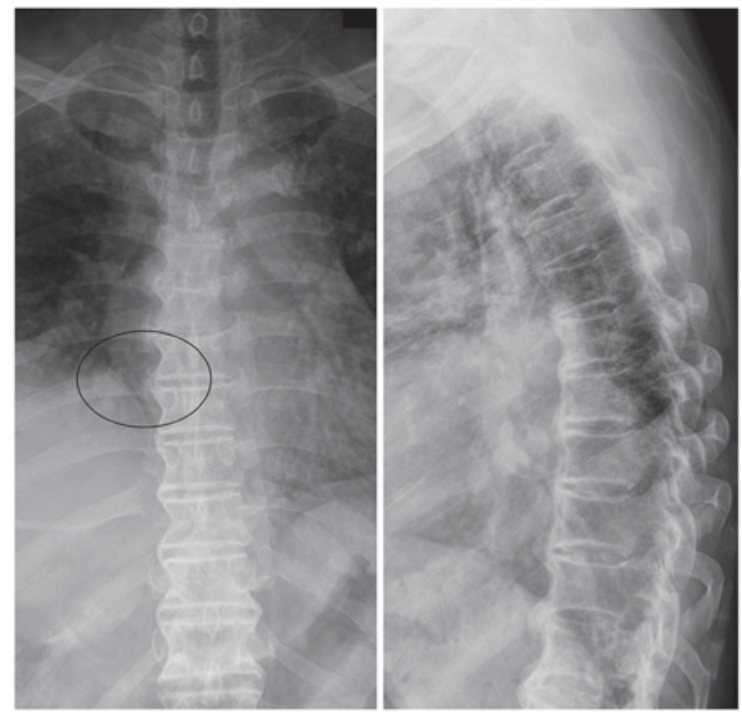

Figure 2. An X-ray on a male patient with hemangioma aged 39 years reveals that the vertebral body is influenced by the lesion, part of the trabeculae of vertebral body is absorbed and part of it shows compensatory thickening and fence-like change.

Evaluation methods. The imaging results were analyzed by two senior imaging diagnosis physicians (with more than 10 -year working experience). When the results obtained by them were inconsistent, they would reach a common conclusion by discussion. Sensitivity (Sen), specificity (Spe), positive predictive value $\left(\mathrm{PV}^{+}\right)$, negative predictive value $\left(\mathrm{PV}^{-}\right)$and accuracy (Acc) were compared among the three examination methods using the pathological results as the gold standard.

Statistical analysis. Four parameters (including true positive, false positive, true negative and false negative) were established for X-ray, CT and MRI diagnosis tests, respectively, using the pathological results as the gold standard, and indicators of diagnostic efficacy were calculated. Positive referred to malignant tumors, while negative referred to benign tumors. Statistical Product and Service Solutions (SPSS) 19.0 (SPSS Inc., Chicago, IL, USA) was adopted for statistics. A paired $\chi^{2}$ test was performed to analyze the difference and consistency of accuracy of X-ray, CT and MRI examinations in diagnosing primary spinal osseous tumors. ANOVA was used for comparison between multiple groups and the post hoc test was LSD test. $\mathrm{P}<0.05$ was considered to indicate a statistically significant difference.

\section{Results}

Comparison of imaging features of different examination methods. CT scan had obvious advantages in diagnosing tumors such as lesion calcification and osteolytic destruction (Fig. 1); X-ray showed better contrast and clarity of the spine, and bone structure could be seen clearly by this method (Fig. 2); MRI was more sensitive to the imaging of the spinal cord, which could be used to discover early tumor lesion based on the change in bone marrow signal (Fig. 3).

Comparison of diagnostic efficacy of different examination methods. X-ray, CT and MRI examination showed that Sen, 

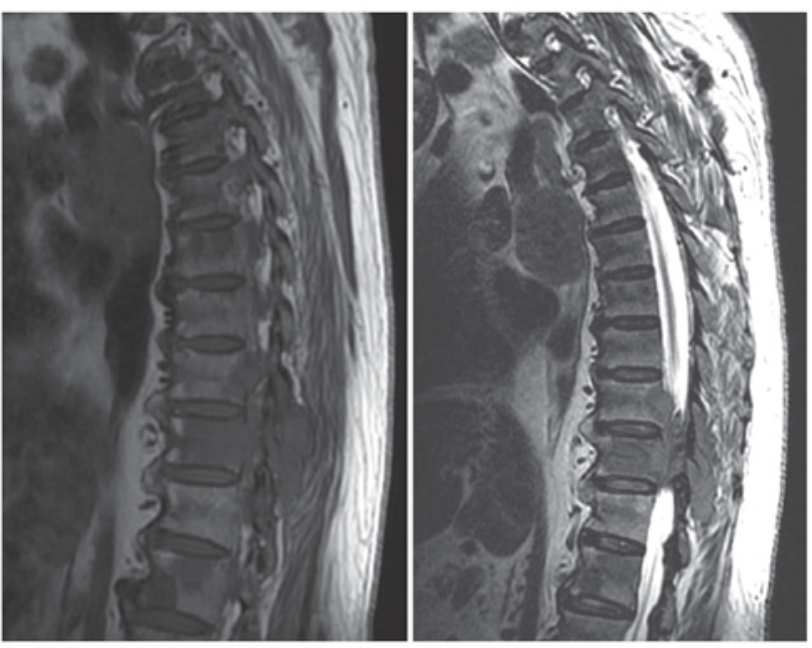

Figure 3. An MRI on a female patient with chondrosarcoma aged 51 years shows that the distribution of density and signal of spinal tumor is not uniform due to the insufficient blood supply in tumor center, and that the sign of 'salt and pepper' is generated.

Table II. Comparison of diagnostic efficacy among X-ray, CT and MRI examination methods $(\%)$.

\begin{tabular}{lccccc}
\hline Examination method & Sen & Spe & Acc & PV $^{+}$ & PV $^{-}$ \\
\hline X-ray & 76.81 & 86.96 & 78.26 & 92.75 & 62.32 \\
CT & 86.96 & 88.41 & 86.96 & 94.20 & 72.46 \\
MRI & $92.75^{\text {a }}$ & 89.86 & $91.30^{\text {a }}$ & 95.65 & $85.51^{\text {a }}$ \\
\hline
\end{tabular}

${ }^{\mathrm{a}} \mathrm{P}<0.05$, comparisons between $\mathrm{X}$-ray and values.

Acc and $\mathrm{PV}^{-}$of the three examination methods in diagnosing spinal osseous tumors were significantly different. MRI examination showed the highest Sen $(\mathrm{P}<0.05)$. Spe and $\mathrm{PV}^{+}$ of the three examination methods had no obvious differences (P>0.05) (Table II).

Diagnostic consistency of MRI and CT with pathological results. The diagnostic results of primary spinal osseous tumors obtained with MRI examination and those obtained with CT scan were compared with the pathological results, respectively, which showed that 45 patients had consistent results with the pathological results by the two methods, and that 19 patients had inconsistent results with the pathological results by the two methods. The two methods had relatively high consistency in diagnosing primary spinal osseous tumors $(\kappa$-value $=0.72)($ Table III $)$.

Diagnostic consistency of CT and X-ray with pathological results. The diagnostic results of primary spinal osseous tumors obtained with CT scan and those obtained with X-ray were compared with the pathological results, which showed that 42 patients had consistent results with the pathological results by the two methods, and that 12 patients had inconsistent results with the pathological results by the two methods. The two methods had relatively low consistency in diagnosing primary spinal osseous tumors $(\kappa$-value $=0.47)($ Table IV).
Table III. Comparison between MRI examination and CT scan (n).

\begin{tabular}{lccc}
\hline $\begin{array}{l}\text { Results obtained } \\
\text { with MRI } \\
\text { examination vs. } \\
\text { pathological results }\end{array}$ & \multicolumn{2}{c}{$\begin{array}{c}\text { Results obtained with CT } \\
\text { scan vs. pathological results }\end{array}$} & \\
\cline { 2 - 3 } & Consistent & Inconsistent & Total \\
\hline Consistent & 45 & 3 & 48 \\
Inconsistent & 5 & 16 & 21 \\
Total & 50 & 19 & 69 \\
\hline
\end{tabular}

MRI examination and CT scan have no significant difference in diagnosing primary osseous tumors $\left(\chi^{2}=0.816, \mathrm{P}=0.314\right)$. They have relatively high consistency in diagnosing primary spinal osseous tumors. $\kappa$-value $=2 \times(45 \times 16-5 \times 3) /(45+3) \times(3+16)+(45+5) \times(5+16)=0.72$.

Table IV. Comparison between CT scan and X-ray (n).

\begin{tabular}{|c|c|c|c|}
\hline \multirow{2}{*}{$\begin{array}{l}\text { Results obtained } \\
\text { with CT scan } \\
\text { vs. pathological } \\
\text { results }\end{array}$} & \multicolumn{2}{|c|}{$\begin{array}{l}\text { Results obtained with X-ray } \\
\text { vs. pathological results }\end{array}$} & \multirow[b]{2}{*}{ Total } \\
\hline & Consistent & Inconsistent & \\
\hline Consistent & 42 & 11 & 53 \\
\hline Inconsistent & 4 & 12 & 16 \\
\hline Total & 46 & 23 & 69 \\
\hline
\end{tabular}

CT scan and $\mathrm{X}$-ray have significant difference in diagnosing primary osseous tumors $\left(\chi^{2}=5.287, \mathrm{P}=0.032\right)$. They have relatively low consistency in diagnosing primary spinal osseous tumors. $\kappa$-value $=$ $2 \times(42 \times 12-4 \times 11) /(42+11) \times(11+12)+(42+4) \times(4+12)=0.47$.

Table V. Comparison between MRI examination and X-ray (n).

\begin{tabular}{lccc}
\hline $\begin{array}{l}\text { Results obtained } \\
\text { with MRI } \\
\text { examination vs. } \\
\text { pathological results }\end{array}$ & $\begin{array}{l}\text { Results obtained with X-ray } \\
\text { vs. pathological results }\end{array}$ & \\
\cline { 2 - 3 } & Consistent & Inconsistent & Total \\
\hline Consistent & 41 & 13 & 54 \\
Inconsistent & 4 & 11 & 15 \\
Total & 45 & 24 & 69
\end{tabular}

MRI and $X$-ray have significant difference in diagnosing primary osseous tumors $\left(\chi^{2}=56.247, \mathrm{P}=0.025\right)$. They have relatively low consistency in diagnosing primary spinal osseous tumors. $\kappa$-value $=$ $2 \times(41 \times 11-4 \times 13) /(41+13) \times(13+11)+(41+4) \times(4+11)=0.41$.

Diagnostic consistency of MRI and X-ray with pathological results. The diagnostic results of primary spinal osseous tumors obtained with MRI examination and those obtained with X-ray were compared with the pathological results,respectively, which showed that 41 patients had consistent results with the pathological results by the two methods, and that 11 patients 
had inconsistent results with the pathological results by the two methods. The two methods had relatively low consistency in diagnosing primary spinal osseous tumors $(\kappa$-value $=0.41)$ (Table V).

\section{Discussion}

Spinal osseous tumors can occur in any part of the vertebrae, mainly in the thoracic vertebrae, followed by the sacral and lumbar spine, but rarely occur in cervical spine $(5,6)$. They include benign tumors (such as giant cell tumor of bone, osteochondroma, hemangioma and aneurysmal bone cyst) and malignant tumors (such as chordoma, myeloma, chondrosarcoma and Ewing's sarcoma), of which the malignant spinal tumor frequently occurs in the middle-aged and the elderly, while the benign tumors are mainly concentrated in the young people (7). Surgical treatments such as radiotherapy, chemotherapy and laminectomy are usually adopted in clinic for the treatment of spinal osseous tumors. With the continuous development of imaging and related medical technology, early detection and surgical treatment have become indispensable treatment methods (8).

X-ray plain film can be available to find most of the vertebral tumors. Its diagnostic principle is that it has relatively high sensitivity and relatively high spatial resolution in imaging bone tissues, and can clearly show the size and the structure of the bone as well as the destruction on the bones in the skeleton and the periosteal reaction based on the change in bone mineral density caused by the change in the concentration of calcium in the spine $(4,9)$. However, it cannot be used for the examination and analysis of cartilage lesions due to its limitations, and the adnexal tumors and those under the adnexa are easy to be omitted. The examination is the most basic method for the diagnosis of tumors (10). Vertebral tumors can be diagnosed with CT examination by the discovery of calcified lesions (11). The imaging of spinal tumors with MRI examination is characterized by clear lesion site and the generation of 'salt and pepper' sign. In addition to displaying the change in morphology of the tumors, it can also reflect the change in signal strength of the lesion, thus effectively improving the accuracy of diagnosis (12).

With the continuous development of imaging diagnosis, CT and MRI techniques have been commonly used in the diagnosis of skeletal diseases, and the early diagnosis of bone tumors and the confirmation of benign lesions are improved. Imaging diagnosis can be used to know the extent of the lesion, and understand whether the surrounding tissues are changed, which is of great significance for clinical diagnosis and treatment (13). The results of this study showed that Sen, Acc and $\mathrm{PV}^{-}$of the three examination methods had no obvious differences in the diagnosis of spinal osseous tumors. MRI examination showed the highest Sen $(\mathrm{P}<0.05)$. Spe and $\mathrm{PV}^{+}$ of the three examination methods had no obvious differences $(\mathrm{P}>0.05)$. This is because the displaying of soft tissue mass of spinal osseous tumors on the X-ray plain film is sometimes unobvious even though X-ray examination is easy to be performed with strong integrity (14). However, CT examination is more sensitive to the resolution of calcified lesions, especially lobular calcified lesions. For example, chondrosarcoma often occurs in the sacrococcygeal vertebra and the thoracic vertebra, and the soft-tissue mass will be ring-arc shaped, and represented as osteolytic destruction, which can be displayed clearly by enhanced CT scan (15). With the progress of the disease, osteolytic destruction is aggravated, the tumor shows invasive growth, and the blood supply in the center of the mass is insufficient. Upon MRI examination, the distribution of density and signal of the tumor is not uniform, most of T1WI show relatively low signal, while T2WI shows relatively high mixed signals. Moreover, MRI has the advantages of direct multi-plane imaging and high resolution. It is more accurate than X-ray plain film and CT scan in displaying tumors inside and outside the spine (16).

The results of this study showed that MRI and CT had no obvious difference in diagnosing primary spinal osseous tumors $(\mathrm{P}>0.05)$, and they had relatively high consistency in diagnosing primary spinal osseous tumors $(\kappa=0.72)$; $\mathrm{CT}$ and $\mathrm{X}$-ray film had obvious difference in diagnosing primary spinal osseous tumors $(\mathrm{P}<0.05)$, and they had relatively low consistency in diagnosing primary spinal osseous tumors $(\kappa=0.47)$; MRI and X-ray film had obvious difference in diagnosing primary spinal osseous tumors $(\mathrm{P}<0.05)$, and they had relatively low consistency in diagnosing primary spinal osseous tumors $(\kappa=0.41)$. This is because $\mathrm{X}$-ray plain film mainly relies on the display of change in bone mineral density to diagnose bone tumors, while it has relatively low sensitivity in diagnosing tumors such as hemangioma and chondrosarcoma. CT scan has the advantages of rapid scanning speed and clear images. With the rapid development of medical imaging technology, CT scan has grown from the past 4 rows to 8, 16 and 64 rows, and even higher (17). Multi-row CT scan reduces the scanning time, and its interference by the motion artifact is reduced. Moreover, $\mathrm{Z}$-axis resolution is higher, which is conducive to showing tiny lesions clearly, thus realizing early detection of primary spinal osseous tumors, and effectively avoiding the shortcomings of X-ray plain film. However, CT scan still has some defects. For example, it is not sensitive to the micro-periosteal reaction of the spinal osseous tumors, which causes missed diagnosis or misdiagnosis. In addition, the radiation generated during examination is harmful to the patient, and frequent examinations in a short term are not appropriate (18). MRI is an imaging technology developed on the basis of magnetic resonance phenomenon. It is a non-radioactive examination with the advantages of high spatial resolution and multi-directional and multi-sequential imaging. It has relatively high sensitivity for the examination in a local range, and can determine the scope of the lesions in the parts involved by the tumors. Furthermore, it has relatively high resolution for tissues, and is more sensitive for displaying the change in osteogenesis of osteoblastoma (19). The scope of the lesion in the spinal cord can be shown effectively and the anatomical structure of the bone with tumor can be reflected clearly through T1WI sequence examination. The correlation of the lesion with blood vessels, nerves and muscles at the adjacent vertebral segment can be shown through T2WI sequence examination, thus effectively judging the violation of the lesion on the surrounding soft tissues. Therefore, MRI examination has relatively high consistency with pathological examination (20).

In conclusion, X-ray, CT and MRI examinations have their own advantages in diagnosing primary spinal osseous tumors. 
Comparatively speaking, CT and MRI examinations have higher accuracy, and they are more conducive to improving the accuracy in the diagnosis of primary spinal osseous tumors. Thus, they have higher clinical values.

\section{Acknowledgements}

Not applicable.

\section{Funding}

No funding was received.

\section{Availability of data and materials}

The datasets used and/or analyzed during the present study are available from the corresponding author on reasonable request.

\section{Authors' contributions}

LY contributed to the planning and implementation of the experiments. SZ and RG wrote the manuscript and analyzed general patient data. SZ was responsible for X-ray examination. MW and LY helped with CT examination. CP contributed to MRI examination. All authors read and approved the final manuscript.

\section{Ethics approval and consent to participate}

The study was approved by the Ethics Committee of Nankai Hospital (Tianjin, China). Signed written informed consents were obtained from the patients or the guardians.

\section{Patient consent for publication}

Not applicable.

\section{Competing interests}

The authors declare that they have no competing interests.

\section{References}

1. Guzik G: Current incidence of different morphological types of malignant metastases to the spine based on magnetic resonance imaging. Ortop Traumatol Rehabil 19: 137-144, 2017.

2. Gupta PK, Misra S, Verma R, Soni N, Lamin JC, Mishra RK, Behari S and Kumar S: Primary intradural cervical spine melanocytoma: A rare tumor and review of literature. Neurol India 65: 653-657, 2017.

3. Yang P, Lin J, Liu H, Shen H and Yang HL: Primary bone mantle cell lymphomas with multiple vertebral compression fractures: A case report. Oncol Lett 13: 1288-1292, 2017.

4. Konovalov NA, Nazarenko AG, Asyutin DS, Onoprienko RA, Korolishin VA, Cherkiev IU, Martynova MA, Zakirov BA, Timonin SY and Kosyr'kova AV: The use of intraoperative neuroimaging tools and a navigation system in surgical treatment of primary and metastatic tumors of the spine. Vopr Neirokhir 80: 5-14, 2016 (In Russian).
5. Zhou Z, Wang X, Wu Z, Huang W and Xiao J: Epidemiological characteristics of primary spinal osseous tumors in Eastern China. World J Surg Oncol 15: 73, 2017.

6. Tomasian A, Wallace A, Northrup B, Hillen TJ and Jennings JW: Spine cryoablation: Pain palliation and local tumor control for vertebral metastases. AJNR Am J Neuroradiol 37: 189-195, 2016.

7. Welte SE, Wiskemann J, Scharhag-Rosenberger F, Förster R, Bostel T, Bruckner T, Schlampp I, Meyerhof E, Sprave T, Nicolay $\mathrm{NH}$, et al: Differentiated resistance training of the paravertebral muscles in patients with unstable spinal bone metastasis under concomitant radiotherapy: Study protocol for a randomized pilot trial. Trials 18: 155, 2017.

8. Sciubba DM, De la Garza Ramos R, Goodwin CR, Xu R, Bydon A, Witham TF, Gokaslan ZL and Wolinsky JP: Total en bloc spondylectomy for locally aggressive and primary malignant tumors of the lumbar spine. Eur Spine J 25: 4080-4087, 2016.

9. Boriani S, Gasbarrini A, Bandiera S, Ghermandi R and Lador R: Predictors for surgical complications of en bloc resections in the spine: Review of 220 cases treated by the same team. Eur Spine J 25: 3932-3941, 2016.

10. Kumar N, Zaw AS, Khoo BL, Nandi S, Lai Z, Singh G, Lim CT and Thiery JP: Intraoperative cell salvage in metastatic spine tumour surgery reduces potential for reinfusion of viable cancer cells. Eur Spine J 25: 4008-4015, 2016.

11. Tamam MÖ, Tamam C, Yıldırım D, Mülazımoğlu M and Kamal 1 G: Intramedullary spinal cord metastasis from malignant mesenchymal tumor: Detection with FDG-PET/CT. Mol Imaging Radionucl Ther 24 (Suppl 1): 22-24, 2015.

12. Monserrate A, Zussman B, Ozpinar A, Niranjan A, Flickinger JC and Gerszten PC: Stereotactic radiosurgery for intradural spine tumors using cone-beam CT image guidance. Neurosurg Focus 42: E11, 2017.

13. Klinaki I, Al-Nahhas A, Soneji N and Win Z: 68Ga DOTATATE PET/CT uptake in spinal lesions and MRI correlation on a patient with neuroendocrine tumor: Potential pitfalls. Clin Nucl Med 38: e449-e453, 2013.

14. Kobayashi K, Imagama S, Ando K, Hida T, Ito K, Tsushima M, Ishikawa Y, Matsumoto A, Morozumi M, Tanaka S, et al: Contrast MRI findings for spinal schwannoma as predictors of tumor proliferation and motor status. Spine 42: E150-E155, 2017.

15. Mechl M, Šprláková-Puková A and Keřkovský M: CT and MRI of spinal tumors - Review and differential diagnosis of most common abnormalities. Cesk Radiol 66: 369-378, 2012.

16. Tarnoki DL, Tarnoki AD, Ohlmann-Knafo S and Pickuth D: Pattern of tumour spread of common primary tumours as seen on magnetic resonance imaging. Pathol Oncol Res 22: 79-85, 2016.

17. Chou CT, Chen RC, Lin WC, Ko CJ, Chen CB and Chen YL: Prediction of microvascular invasion of hepatocellular carcinoma: Preoperative CT and histopathologic correlation. AJR Am J Roentgenol 203: W253-W259, 2014.

18. Sahinarslan A, Erbas G, Kocaman SA, Bas D, Akyel A, Karaer D, Ergun MA, Arac M and Boyaci B: Comparison of radiationinduced damage between CT angiography and conventional coronary angiography. Acta Cardiol 68: 291-297, 2013.

19. Ganten MK, Schuessler M, Bäuerle T, Muenter M, Schlemmer HP, Jensen A, Brand K, Dueck M, Dinkel J, Kopp-Schneider A, et al: The role of perfusion effects in monitoring of chemoradiotherapy of rectal carcinoma using diffusion-weighted imaging. Cancer Imaging 13: 548-556, 2013.

20. D'Andrea K, Dreyer J and Fahim DK: Utility of preoperative magnetic resonance imaging coregistered with intraoperative computed tomographic scan for the resection of complex tumors of the spine. World Neurosurg 84: 1804-1815, 2015.

This work is licensed under a Creative Commons Attribution-NonCommercial-NoDerivatives 4.0 International (CC BY-NC-ND 4.0) License. 